\title{
Bifurcation scenarios in helical buckling of slender rods using new FE
}

\author{
Marcin Kapitaniak $^{\mathrm{a}}$, Vahid Vaziri ${ }^{\mathrm{a}}$, Marian Wiercigroch ${ }^{\mathrm{a}, *}$ \\ ${ }^{a}$ Centre for Applied Dynamics Research, School of Engineering, University of Aberdeen, Aberdeen AB24 3UE, UK
}

\begin{abstract}
We develop a new Finite Element to accurately model twisting of rods and capture the bifurcation scenarios leading to helical buckling and various further post-buckling states. Since standard nonlinear beam elements do not account for nonlinearities in torsional modes, as well as for coupling between axial, lateral and torsional modes, we derive a new beam element, which allows us to describe complex helical buckling bifurcation scenarios of a rod subjected to a twisting load. The formulated beam element is systematically tested to assess its predictive capabilities in determining critical torsional buckling loads and its sensitivity to a number of elements used. Once the model is validated against commercial FE software (ABAQUS), we focus our attention on computing bifurcation scenarios to observe various complex helical configurations and transitions between them. The analysis reveals co-existence between helices with multiple loops for certain values of twisting load. Additionally, we trace the transition onsets between stable helical configurations. The developed FE can be applied to study complex buckling mechanics of engineering and biological structures.
\end{abstract}

Keywords: Helical buckling, bifurcations, twisted rods, finite element, post-buckling configuration.

\section{Introduction}

Helical buckling of long thin rods represents an active field of research, that has a variety of practical applications ranging from engineering to chemistry. In terms of engineering applications the theory of helical buckling can be widely applied to study behaviour of underwater cables [1] as well as drill-strings and coil tubing used by energy industries [2-4]. In chemistry and biology applications, this would involve extensive studies on equilibrium configurations of polymers, organic fibers and $\mathrm{DNA}[1,5,6]$. Helical buckling still poses a significant modelling challenge, which has been tackled in the past using analytical approaches [5, 7-11] that allowed to identify various post-buckling helical modes in long twisted and stretched/compressed rods. As a torque acting on a rod increases, often a trivial undeflected solution

\footnotetext{
${ }^{*}$ Corresponding author.

Email addresses: marcin.kapitaniak@abdn.ac.uk (Marcin Kapitaniak), vahid.vaziri@abdn.ac.uk (Vahid Vaziri), m.wiercigroch@abdn.ac.uk (Marian Wiercigroch)
} 
becomes unstable and bifurcates, leading to writhing of the rod, with a number of helical loops increasing. Nayfeh \& Emam [11] investigated the post-buckling configurations (and their stability) for beams under axial loading, by solving dynamic equations of motion, taking into account geometric nonlinearities. A similar approach was adopted by Liu \& Xue [10], who studied stability of the helical equilibrium of thin elastic rods, taking into account shear deformation. Miyazaki \& Kondo [9] focussed on post-buckling behaviour of an elastic beam subjected to end twist and uniaxial end-shortening, which in turn allowed to investigate phenomena of kink formations and snap-through behaviours, associated with secondary bifurcations. A particular attention was given to the configurations when an elastic beam contacts itself, forming a kink. Liu \& Zu [5] investigated analitically the helical equilibrium and its stability for a thin elastic rod of noncircular cross-sections, under axial force and torque. For this purpose the Kirchoff theory was applied to derive a stability condition for the helical equilibrium. Additionally, the authors observed that each helical equilibrium corresponds to a singular point and that the region of instability of the particular equilibrium is heavily related to the cross-section of the rod, which expands as the assymetry of the cross-section grows.

Details of buckling and various post-buckling configurations can be useful in engineering problems, allowing for the design of beams with nonstandard cross-sections, that are more resistant to buckling. An example of that is given by Braun [12], who considers a rotating compressed rod and performs an optimisation studies to find a shape of the rod that can maintain stability against buckling. The authors took into account shear and extensibility of the rod and obtained analytical solutions allowing to study influence of a rod shape on the buckling characteristics.

Another way to tackle the modelling challenges of analysing helical buckling and its post-buckling configurations is to turn to the Finite Element (FE) approach. For basic calculations, like predicting critical buckling modes and basic post buckling analysis, commercial FE softwares (Abaqus, Ansys, LSDyna and others) provide a convenient computational tool. Hovewer, analysis using this approach can be costly in terms of computational time and resources required (in particular CPU and disc space). Moreover, the user has a limited access to the mathematical formulations behind various $\mathrm{FE}$ elements that are available in the commercial software. Due to this fact, quite often a large number of elements is required to observe a particular phenomenon, resulting in long computational time. This drawback can be addressed, if a custom made FE is developed to just capture the phenomena under investigation. By creating a such element, the issue of accuracy and computational time can be greatly improved, by FE elements required when compared to the number needed using a commercial software. An example of this approach has been implemented by Stoykov \& Ribeiro [13], who derived an FE beam element 
for a rectangular cross-section, taking into account geometric nonlinearites and warping. They investigated a nonlinear coupling between bending and torsion and compared differences arising from using Euler-Bernouli and Timoshenko beams. Application of this approach to study helical buckling of thin long rods has been quite limited. For example, Barnes et al. [14] derive a 3D beam element taking into account torsion and transverse bending of curved beams, utilising dynamic relaxation method to validate the element for two test cases of a circular arc. This was done by analysing stability and accuracy of the proposed method and comparing the results to the predictions obtained from more complex FE formulations. Sapountzakis \& Makos [15] constructed a 3D beam element with 7 DOFs per node including both shearing and warping deformations and demonstrated the importance of their inclusion when analysing thick wall cross-sections. Yang \& McGuire [16] investigated the inclusion of nonuniform torsion into the geometric stiffness matrix of a beam element. This was done following principle of virtual displacements and Langangian approach, through adding the warping degree-of-freedom to the standard two node beam element, thereby creating an element with 7 DOFs per node. Meier et al. [17] proposed a locking-free geometrically exact FE formulation based on Kirchoff theory of thin beams, that incorporates axial, lateral and torsional deformations in geometrically nonlinear regimes. Additionally, the authors considered various reduced formulations for specific cases, which provided accurate results and improved computational efficiency. Sen \& Awtar [18] derived a nonlinear beam element with a uniform and symmetric cross-section, that considers geometric nonlinearities due to flexure. The governing equations were derived from the principle of virtual work and the results were verified against FE software for various load and displacement ranges. In summary as shown in a brief overview above, it is a common practice to construct special FE elements that are fine tuned to capture the phenomena under consideration. This approach provides a necessary physical insight into elements capabilities, which in turn allows their optimisation with regards to two main criteria of accuracy and computational cost.

One of the obvious research areas, that would benefit from in-depth studies of helical buckling of rods, is the field of drill-string dynamics, which basically focuses on analysing a long, flexible rod constrained inside a cylinder. Most of the studies in this field involving FE modelling, have neglected the influence of helical buckling on the drill-string behaviour alltogether. Normally, when purposely derived FE elements are applied to model drill-string dynamics, different variations of linear and nonlinear beam elements are used, without taking into account all nonlinearities arising from coupling of the torsional and axial or lateral DOFs. The main focus of previous studies was on inclusion of basic couplings between DOFs [1921], gyroscopic effects [22], gravity [23], uncertaintities in drill-bit and rock interactions [24], the contacts with a bore-hole ([25]) as well as fluid and drill-string interactions [26, 27]. 
In this paper we introduce a modified Euler-Bernoulli two node beam element to study post-buckling bifurcations of twisted rods. The model developed in this paper can be added to the FE library to be used when helical buckling plays an important role. The paper is organised as follows. In Section 2, a detailed derivation of the nonlinear FE beam element coupling axial, lateral and torsional DOFs is presented. Subsequently in Section 3, a validation of the proposed method is conducted to assess predictive capabilities of the model. These results are then compared with the predictions from the commercial FE software (ABAQUS), revealing an excellent agreement. Additionally, a further study to verify sensitivity of the predicted critical buckling load to number of FE elements is conducted. In Section 4, we investigate the post-buckling scenarios of a rod subjected to an end torque, allowing to trace precisely the transitions between various helical configurations as well as co-existence of two different helical configurations. Moreover, we follow the evolution of the helical configurations of the rod and pinpoint the onset to the transitions between various states. Section 5 draws conclusions and gives a summary of the most important outcomes of this work with suggestions for the future work.

\section{Nonlinear beam element}

In order to gain a deeper insight into the mechanics of helical buckling of rods by applying FEM, it is vital to use efficient and robust finite elements, that can precisely capture coupling effects between twisting and bending DOFs. This can be achieved by a modification of 3D large deflection Euler-Bernoulli or Timoshenko beam elements [28], which is based on including appropriate nonlinear strain components [29].

Let us consider a 3D Euler-Bernoulli circular beam element, with two nodes and six degrees-of-freedom per node (3 displacements $x, y, z$ and 3 rotations $\left.\zeta_{x}, \zeta_{y}, \zeta_{z}\right)$, as depicted in Fig. 1(d). We assume isotropic beams with axisymmetric cross-sections (e.g. circular, square etc.), which means that warping effects are not present. As the first step in derivation of the geometric nonlinear stiffness of a beam, it is required to describe the displacement field of the beam $\left(Q_{1}, Q_{2}, Q_{3}\right)$ along axes $x, y, z$. Figs 1(a)-(c) present schematics depicting the displacements of the beam (continuous line) from the undeformed configuration (dashed line). Looking at the displacements of the points $P_{1}, P_{2}, P_{3}, P_{4}$, where " " denotes the deformed configuration, one can easily write down the displacement fields as: 


$$
\begin{aligned}
Q_{1} & =u-u_{x 1}-u_{x 2}=u-y \tan \theta_{z}+z \tan \theta_{y} \approx u-z \theta_{y}-y \theta_{z}=u-z \frac{\partial w}{\partial x}-y \frac{\partial v}{\partial x}, \\
Q_{2} & =v-y\left(1-\cos \theta_{x}\right)-z \sin \theta_{x}, \\
Q_{3} & =w-z\left(1-\cos \theta_{x}\right)+y \sin \theta_{x},
\end{aligned}
$$

which involve combinations of displacements $u, v, w$ along $x, y, z$ axes and rotations $\theta_{x}, \theta_{y}, \theta_{z}$ around $x, y$, $z$ axes. This is a straightforward procedure, that is well explained in FE textbooks (see e.g. [28, 30, 31]). The rotations $\theta_{y}, \theta_{z}$ are approximated as $\theta_{y}=-\frac{\partial v}{\partial x}, \theta_{z}=\frac{\partial w}{\partial x}$, while sine and cosine terms involving $\theta_{x}$ are not expanded at this point following approach suggested by Stoykov and Ribeiro [13].
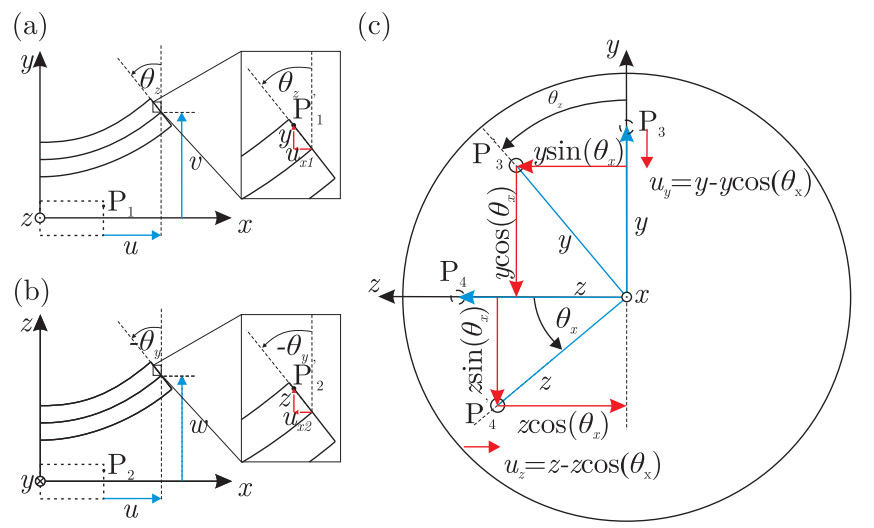

(d)
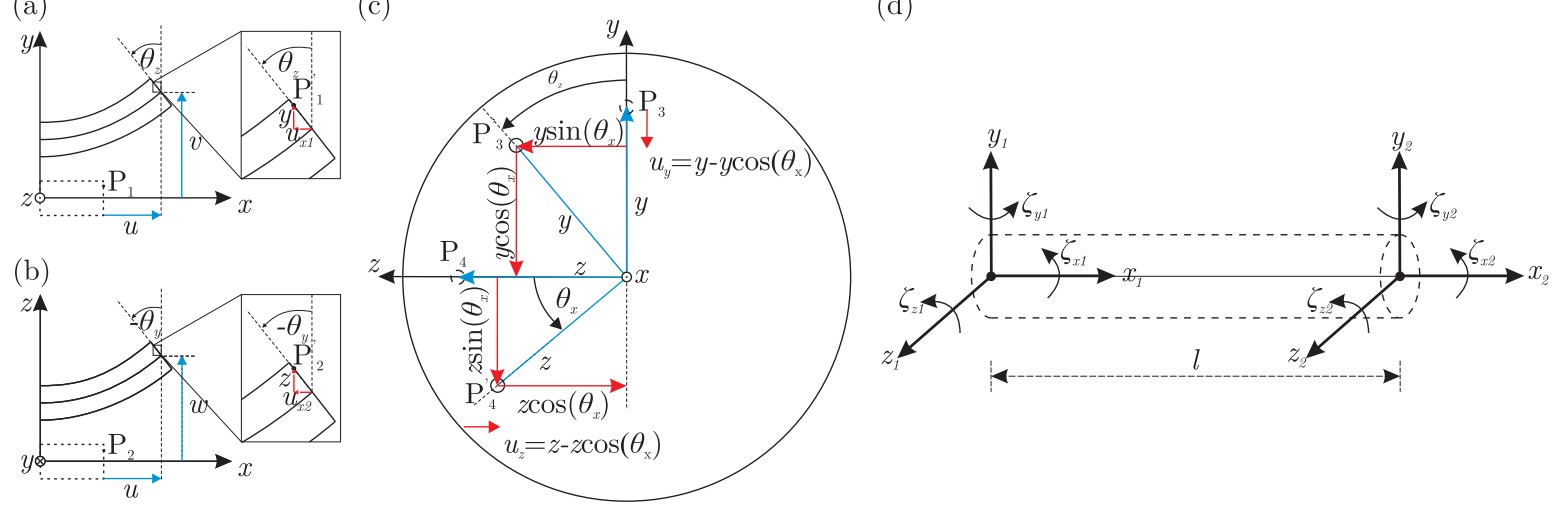

Figure 1: (a) Schematics depicting deformations of the beam (marked in continuous lines) from the undeformed configuration (marked in dashed line). (d) Two node axisymmetric 3D beam element.

In order to derive a stiffness matrix for a beam element, one needs to calculate the strain energy, which for isotropic materials can be expressed as

$$
U_{i}=\frac{1}{2} \int\left(E\left(\epsilon_{x x}^{2}+\epsilon_{y y}^{2}+\epsilon_{z z}^{2}\right)+G\left(\epsilon_{x y}^{2}+\epsilon_{y z}^{2}+\epsilon_{z x}^{2}\right)\right) d V
$$

Assuming, that a cross-section of the beam does not change during the deformation $\left(\epsilon_{y y}=0, \epsilon_{z z}=\right.$ $0, \epsilon_{y z}=0$, ), the strain energy during deformation can be simplified to

$$
U_{i}=U_{x x}+U_{x y z x}=\frac{1}{2} \int E \epsilon_{x x}^{2} d V+\frac{1}{2} \int G\left(\epsilon_{x y}^{2}+\epsilon_{z x}^{2}\right) d V
$$

where $U_{x x}$ and $U_{x y z x}$ are axial and shear strain energies, while the axial and shear strains are derived from Green's strain tensor as 


$$
\begin{aligned}
& \epsilon_{x x}=\frac{\partial Q_{1}}{\partial x}+\frac{1}{2}\left(\left(\frac{\partial Q_{1}}{\partial x}\right)^{2}+\left(\frac{\partial Q_{2}}{\partial x}\right)^{2}+\left(\frac{\partial Q_{3}}{\partial x}\right)^{2}\right) \\
& \epsilon_{x y}=\frac{\partial Q_{1}}{\partial y}+\frac{\partial Q_{2}}{\partial x}+\left(\frac{\partial Q_{1}}{\partial x} \frac{\partial Q_{1}}{\partial y}+\frac{\partial Q_{2}}{\partial x} \frac{\partial Q_{2}}{\partial y}+\frac{\partial Q_{3}}{\partial x} \frac{\partial Q_{3}}{\partial y}\right), \\
& \epsilon_{z x}=\frac{\partial Q_{1}}{\partial z}+\frac{\partial Q_{3}}{\partial x}+\left(\frac{\partial Q_{1}}{\partial x} \frac{\partial Q_{1}}{\partial z}+\frac{\partial Q_{2}}{\partial x} \frac{\partial Q_{2}}{\partial z}+\frac{\partial Q_{3}}{\partial x} \frac{\partial Q_{3}}{\partial z}\right) .
\end{aligned}
$$

Note, that the common approximations $\sin \theta_{x} \approx \theta_{x}$ and $\cos \theta_{x} \approx 1$ are applied at this point after calculating strains, rather than following the typical approach in the displacement Equations (1), as suggested originally by Stoykov and Ribeiro [13]. This improves the precision, reduces number of high order terms and leads to the following expressions, where for simplicity we changed notation, $\frac{\partial}{\partial x}=\left({ }^{\prime}\right)^{\prime}$,

$$
\begin{aligned}
& \epsilon_{x x}=u^{\prime}-w^{\prime \prime} z-v^{\prime \prime} y-u^{\prime} w^{\prime \prime} z-u^{\prime} v^{\prime \prime} y+\frac{1}{2} w^{\prime \prime 2} z^{2}+\frac{1}{2} v^{\prime \prime 2} y^{2}+\frac{1}{2} u^{\prime 2}+\frac{1}{2} w^{\prime 2}+\frac{1}{2} v^{\prime 2}, \\
& \epsilon_{x y}=-z \theta_{x}^{\prime}-v^{\prime} u^{\prime}+v^{\prime} w^{\prime \prime} z+v^{\prime} v^{\prime \prime} y+\theta_{x} w^{\prime}, \\
& \epsilon_{z x}=y \theta_{x}^{\prime}-w^{\prime} u^{\prime}+w^{\prime} w^{\prime \prime} z+w^{\prime} v^{\prime \prime} y-\theta_{x} v^{\prime} .
\end{aligned}
$$

It is important to reiterate that in the above equations, the terms associated with warping, which are not present for the case of circular and other axisymmetric cross-sections, are omitted. Using the above Equations (7)-(9), one can calculate the axial strain energy as

$$
\begin{array}{r}
U_{x x}=\frac{E}{2} \int_{0}^{l} \int_{A}\left[\left(1+\frac{1}{2}{w^{\prime}}^{2}+\frac{1}{2} v^{\prime 2}+\frac{3}{2}{u^{\prime}}^{2}+3 u^{\prime}\right)\left(w^{\prime \prime 2} z^{2}+v^{\prime \prime 2} y^{2}\right)+u^{\prime 2}\left(1+\frac{1}{2} v^{\prime 2}+\frac{1}{2}{w^{\prime}}^{2}\right)+\right. \\
\left.+u^{\prime}\left({w^{\prime 2}}^{\prime 2}+v^{\prime 2}\right)+u^{\prime 3}+\frac{1}{2} v^{\prime 2} w^{\prime 2}+\frac{1}{4} w^{\prime 4}+\frac{1}{4} v^{\prime 4}+\frac{1}{4} u^{\prime 4}\right] d x d A
\end{array}
$$

as well as shear strain energy

$$
\begin{array}{r}
U_{y x z x}=\frac{G}{2} \int_{0}^{l} \int_{A}\left[\left({\theta_{x}^{\prime}}^{2}+2 v^{\prime \prime} \theta_{x}^{\prime} w^{\prime}+{v^{\prime \prime}}^{2}\left({w^{\prime}}^{2}+{v^{\prime}}^{2}\right)\right) y^{2}+\right. \\
\left.+\left({\theta_{x}^{\prime 2}}^{2}-2 w^{\prime \prime} \theta_{x}^{\prime} v^{\prime}+w^{\prime \prime 2}\left({w^{\prime 2}}^{2}+{v^{\prime}}^{2}\right)\right) z^{2}+{u^{\prime}}^{2}\left({w^{\prime 2}}^{2}+{v^{\prime}}^{2}\right)\right] d x d A .
\end{array}
$$

The higher order terms $(\geqslant 2)$ involving $\frac{\partial \theta_{x}}{\partial x}$ are omitted from the above equations, as otherwise the 
derived beam element would violate Saint-Venant torsion theory and its twist would not be uniform. A selection process to decide an influence of each removed component has been carefully carried out. The retained terms in the expressions for strain energies provide a geometric nonlinear coupling between axial, lateral and torsional DOFs and their choice will be verified in Section 3. The next step in the derivation process is to choose the shape functions that relate the displacements $u, v, w$ to the nodal displacements $x_{1}, y_{1}, z_{1}, \zeta_{x 1}, \zeta_{y 1}, \zeta_{z 1}, x_{2}, y_{2}, z_{2}, \zeta_{x 2}, \zeta_{y 2}, \zeta_{z 2}$. For this purpose, we use standard polynomial shape functions for Euler-Bernoulli beam [28, 32], shown below

$$
\left[\begin{array}{c}
u \\
v \\
w \\
\theta_{x}
\end{array}\right]=\left[\begin{array}{cccc}
1-\xi & 0 & 0 & \\
0 & 1-3 \xi^{2}+2 \xi^{3} & 0 & \\
0 & 0 & 1-3 \xi^{2}+2 \xi^{3} & \\
0 & 0 & 0 & 1-\xi \\
0 & 0 & \left(-\xi+2 \xi^{2}-\xi^{3}\right) l & \\
0 & \left(\xi-2 \xi^{2}+\xi^{3}\right) l & 0 & \\
\xi & 0 & 0 & \\
0 & 3 \xi^{2}-2 \xi^{3} & 0 & \\
0 & 0 & 3 \xi^{2}-2 \xi^{3} & \\
0 & 0 & 0 & \left(\xi^{2}-\xi^{3}\right) l
\end{array}\right]^{\mathrm{T}}\left[\begin{array}{c}
x_{1} \\
y_{1} \\
z_{1} \\
\zeta_{x 1} \\
\zeta_{y 1} \\
\zeta_{z 1} \\
x_{2} \\
y_{2} \\
z_{2} \\
\zeta_{x 2} \\
\zeta_{y 2} \\
\zeta_{z 2}
\end{array}\right],
$$

where $\xi=\frac{x}{l}$. Internal forces generated inside of the element $S_{i}$ due to nodal displacements can be calculated by differentiating the internal strain energy with respect to the nodal displacements $\boldsymbol{u}=\left[x_{1}\right.$, $\left.y_{1}, z_{1}, \zeta_{x 1}, \zeta_{y 1}, \zeta_{z 1}, x_{2}, y_{2}, z_{2}, \zeta_{x 2}, \zeta_{y 2}, \zeta_{z 2}\right]$ as

$$
\left[\begin{array}{c}
S_{1} \\
S_{2} \\
\vdots \\
S_{12}
\end{array}\right]=\left[\begin{array}{c}
\frac{\partial U_{i}}{\partial u_{1}} \\
\frac{\partial U_{i}}{\partial u_{2}} \\
\vdots \\
\frac{\partial U_{i}}{\partial u_{12}}
\end{array}\right]=\left[\boldsymbol{k}_{\boldsymbol{E}}+\boldsymbol{k}_{G}\left(x_{1}, y_{1}, z_{1}, \zeta_{x 1}, \zeta_{y 1}, \zeta_{z 1}, x_{2}, y_{2}, z_{2}, \zeta_{x 2}, \zeta_{y 2}, \zeta_{z 2}\right)\right] \boldsymbol{u}
$$

where $\boldsymbol{k}_{\boldsymbol{G}}\left(x_{1}, y_{1}, z_{1}, \zeta_{x 1}, \zeta_{y 1}, \zeta_{z 1}, x_{2}, y_{2}, z_{2}, \zeta_{x}, \zeta_{y 2}, \zeta_{z 2}\right)$ is the nonlinear geometric stiffness matrix. As the beam has to be in equilibrium, the internal forces $S_{i}$ must be equal to the external forces $\boldsymbol{F}_{\boldsymbol{e}}=$ $\left[F_{x 1}, F_{y 1}, F_{z 1}, M_{x 1}, M_{y 1}, M_{z 1}, F_{x 2}, F_{y 2}, F_{z 2}, M_{x 2}, M_{y 2}, M_{z 2}\right]$ acting on the beam. In this way, we obtain a set of linear and nonlinear stiffness matrices for the 12 DOFs beam element, which will be used in the subsequent sections to assembly the global stiffness matrix that allow us to perform veryfying tests on 
the beam element derived above for various boundary conditions and numbers of elements.

\section{Validation of the beam element}

In order to perform a validation of the derived beam element, we need to ensure that the torsional buckling occurs for the correct loading conditions. For that purpose, let us consider a steel rod ( $E=$ $210.00 \mathrm{GPa}, G=76.92 \mathrm{GPa}$ ) of length, $L=20 \mathrm{~m}$ and circular cross-section of radius $r=0.025 \mathrm{~m}$, supported as shown in Fig. 2 (a). The rod is supported at its both ends, where for the left hand side node (1) all the displacement DOFs $\left(x_{1}, y_{1}, z_{1}\right)$ and rotation around $x\left(\zeta_{x 1}\right)$ axis are fixed, while for the right hand side node (2) it is subjected to an end torque $M_{\text {end }}$ and lateral DOFs are fixed $\left(y_{2}, z_{2}\right)$. Using the linear and nonlinear stiffness matrices derived in Section 2, the rod is then discretized into $N=60$ elements, meaning 61 nodes, each having six DOF per node. The discretized rod is depicted schematically in Fig. 2 (b), which shows the initial straight configuration and its centreline, where boundary conditions described above and shown in panel (a) are applied at nodes (1) and (61) respectively. The boundary conditions are applied following the standard FE protocol of removing corresponding rows and columns from the global stiffness matrix $[28,30]$, assembled for $N$ number of elements (in our case the global stiffness matrix has size $360 \times 360)$. Note, that the deflections of the rod are monitored by computing displacements of its centreline. An example of such a deformed configuration for the analyzed case is given in Fig. 2 (c).

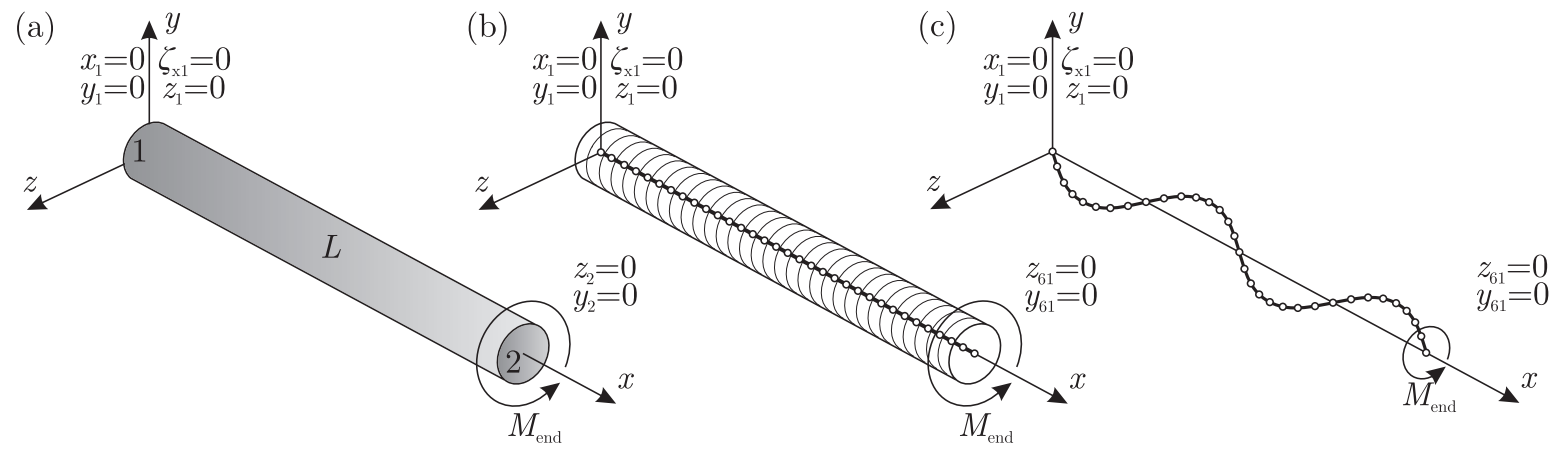

Figure 2: (a) Configuration of the analyzed rod of length $L$, showing the boundary conditions and loading applied at its both ends (node 1: $x_{1}=0, y_{1}=0, z_{1}=0, \zeta_{x 1}=0$, node 2: $y_{2}=0, z_{2}=0$ ), (b) Initial straight configuration of the rod shown in panel (a) discretized into $N=60$ elements (centreline), with same boundary conditions applied at nodes 1 and 61 respectively, (c) Example of the deformed configuration.

\subsection{Validation framework}

As means of verification of the torsional buckling modes, we perform the modal analysis in ABAQUS [33] to determine critical torsional buckling loads, for a rod of the same dimensions and boundary condi- 
tions. These results are then used as a reference to determine the results obtained using the FE element derived in Section 2. In order to compute the critical torsional buckling modes we solve the equilibrium equation between the internal forces and external loading, which can be written in the following form

$$
\left(\boldsymbol{K}_{G L e}+\boldsymbol{K}_{G L g}\right) \boldsymbol{U}=\boldsymbol{F}_{G L e},
$$

where $\boldsymbol{K}_{\boldsymbol{G} \boldsymbol{L} \boldsymbol{e}}$ and $\boldsymbol{K}_{\boldsymbol{G} \boldsymbol{L} \boldsymbol{g}}$ are the linear and nonlinear geometric global stiffness matrices, $\boldsymbol{U}=\left[\zeta_{y 1}, \zeta_{z 1}\right.$, $\left.x_{2}, y_{2}, z_{2}, \zeta_{x 2}, \cdots, x_{361}, \zeta_{x 361}, \zeta_{y 361}, \zeta_{z 361}\right]^{\mathrm{T}}$ is the vector of global coordinates and $\boldsymbol{F}_{\boldsymbol{G} \boldsymbol{L} \boldsymbol{e}}=[0,0,0$, $\left.0,0,0, \cdots, 0, M_{e n d}, 0,0\right]^{\mathrm{T}}$ is the vector of external loading. The assembly of the global matrices is performed in Maple. Eq. 14 describes the static equilibrium of the analyzed rod, while its response to the external loading is computed using the Newton-Raphson iterative method to determine nodal displacements, currently implemented in Matlab. The simulation starts from the straight configuration for a given value of the end torque in the vicinity of the critical buckling load computed in Abaqus, which is shown in second column of Table 1 .

Once the nodal displacements are computed, the end torque is increased in steps of $0.1 \mathrm{Nm}$, while the previous solution is used as a starting point of the computation for the new value of the load. This procedure is repeated until a significant change is the lateral displacements of the rod is observed. For example, to determine the first torsional buckling load, we start the simulation at $M_{\text {end }}=10000 \mathrm{Nm}$ computing the nodal displacements for consecutive steps $\left(\triangle M_{\text {end }}=0.1 \mathrm{Nm}\right)$ until $M_{\text {end }}=20000 \mathrm{Nm}$. This allows to trace precisely the onset to torsional buckling and consequently to determine the value of the first critical torsional buckling load marked as (I). This result is depicted as the orange curves shown in Figs 3 and 4, showing the lateral and torsional displacements of the middle node (node 31). By examining the evolution of the lateral displacement $y_{31}$ shown in Fig. 3(a), it is clear that there is no visible change in the shape until the critical torsional buckling load is reached (marked by the vertical dashed line), when a sudden jump in $y_{31}$ is observed. As $M_{\text {end }}$ increases, so does the lateral displacement, leading to the post-buckling behaviour of the rod, which will be analyzed in more detail in the next section.

Please note, that different variables including displacements along $x$ or $z$ axes or rotations around $y$ or $z$ axes can also be utilized to trace the onset to buckling. In order to compute next critical torsional buckling loads, we repeat the above procedure, always starting the simulation in the vicinity of the expected buckling load and from the straight configuration. The results of the simulation for the II, III, IV, V, and VI torsional buckling modes are shown also in Fig. 3, in purple, cyan, blue, red and green colours, respectively. The exact values of the critical torsional buckling loads (marked by vertical dashed 
Table 1: Comparison between critical torsional buckling loads computed in Abaqus and using model described in Section 2.

\begin{tabular}{|c|c|c|c|}
\hline Buckling mode & Abaqus [Nm] & Nonlinear element [Nm] & Error [\%] \\
\hline \hline I & 15822 & 15814.2 & 0.050 \\
\hline II & 33712 & 33714.4 & 0.007 \\
\hline III & 52858 & 52860.6 & 0.005 \\
\hline IV & 72516 & 72524.7 & 0.010 \\
\hline V & 92396 & 92420.6 & 0.026 \\
\hline VI & 112389 & 112442.5 & 0.047 \\
\hline
\end{tabular}

lines in Fig. 3) are shown in Table 1. It is important to highlight at this point, an excellent agreement between the critical torsional buckling loads predicted using our FE element and the results obtained from Abaqus, which manifests itself in small values of relative errors for each mode. In Fig. 4 we compare a 3D view of the shapes of the rod in each of the considered buckling modes, where the left panel depicts the result from the FE model derived in Section 2, while the right one corresponds to the result computed in Abaqus. Modes I and II have relatively simple shapes, compared to modes III, IV, V and VI. Note, that the number of loops visible corresponds directly to the mode number.
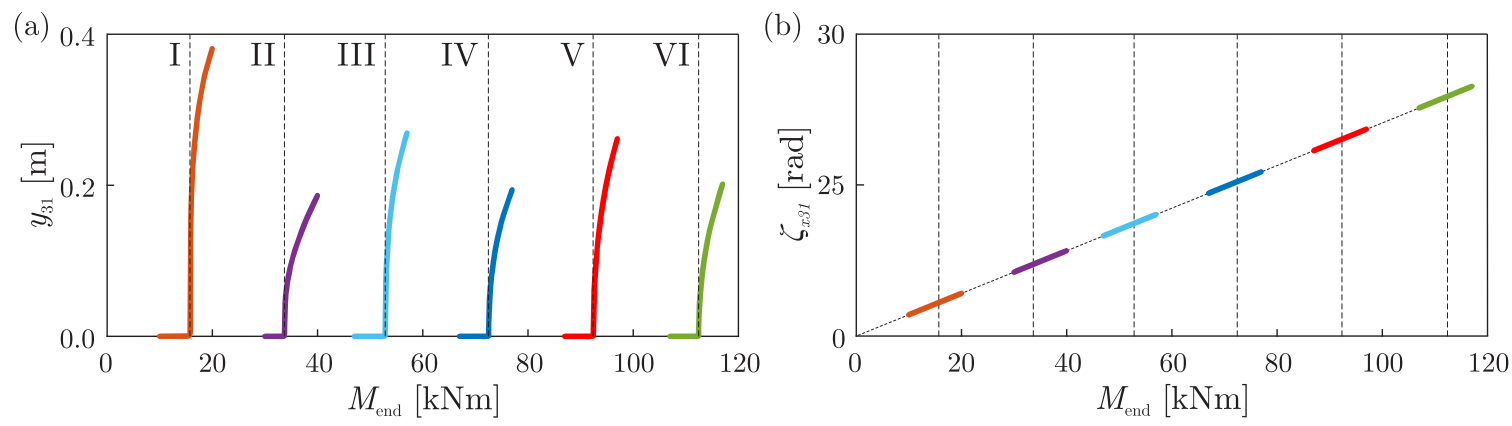

Figure 3: Computation of critical torsional buckling modes using the proposed approach, where (a) depicts the lateral displacement and (b) torsional displacement of the middle node of the rod (31). Vertical dashed lines mark the onset to torsional buckling, which gives us the critical torsional buckling loads (I-VI). 

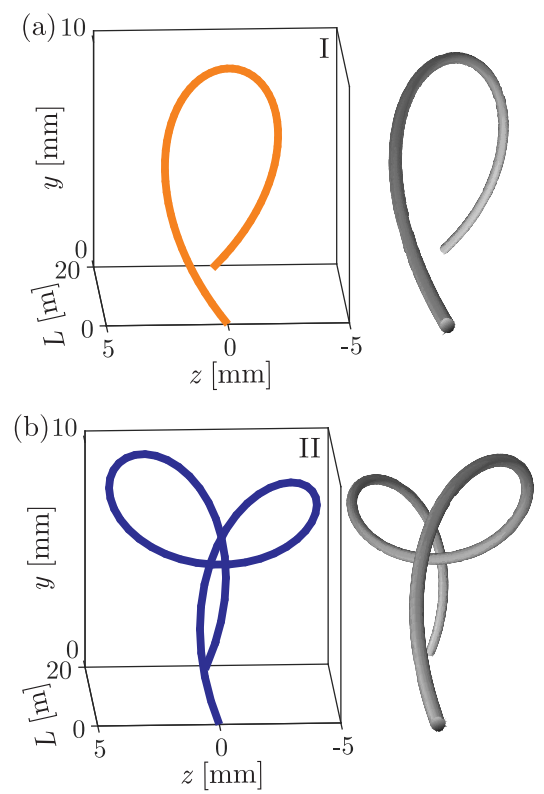
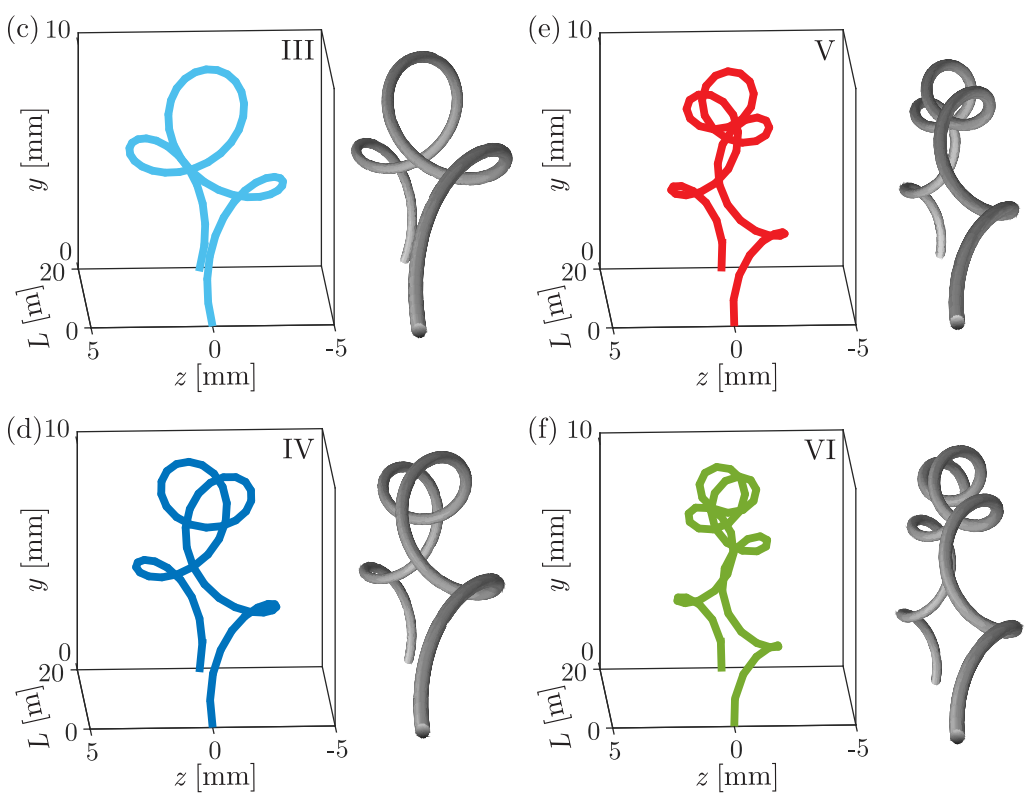

Figure 4: Comparison between the 3D shapes of the analyzed rod computed using the proposed FE element (left panel) and Abaqus (right panel) for critical buckling modes (a) I, (b) II, (c) III, (d), IV, (e) V and (f) VI.

\subsection{Sensitivity to number of elements}

In FE modelling, an element type as well as a number of elements used to discretize the model, play a vital role in the accuracy of the obtained results. In addition, there needs to be a compromise between the accuracy and the computational time. Therefore, it is important to assess the influence of a number of elements on the accuracy in the torsional critical buckling loads predictions. For that purpose, we repeat the simulations described in the previous section for various number of elements from the interval, $N \in(4,60)$. The summary of these calculations is presented in Fig. 5. Looking at the first mode (in orange), we see that for $N=4$, the critical buckling load is slightly off from the value predicted by Abaqus (horizontal dashed line). As $N$ increases, the solution quickly converges to the constant value $(N=8)$, while further increase of $N$ does not affect the solution. Exactly the same trend is present for higher torsional buckling modes (II, III, IV, V and VI marked in purple, cyan, blue, red and green colours), with the only difference lying in the fact that the minimum $N$ increases with the mode number, meaning for example that using $N=4$ one is able to capture just the first mode. To determine complex shapes of the higher modes, which projections on y-z plane are depicted in the left and right panels in Fig. 5, there is a minimum number of elements marked by the left borders of the horizontal sections of curves depicted in the middle panel of Fig. 5. This means that in order to capture all six torsional critical buckling modes one needs to use at least $N=15$ elements (vertical dashed line). All the considered buckling modes I-VI converge to the reference solution for $N=28$, which means that this is the minimum number of elements 
that provide accurate solutions.
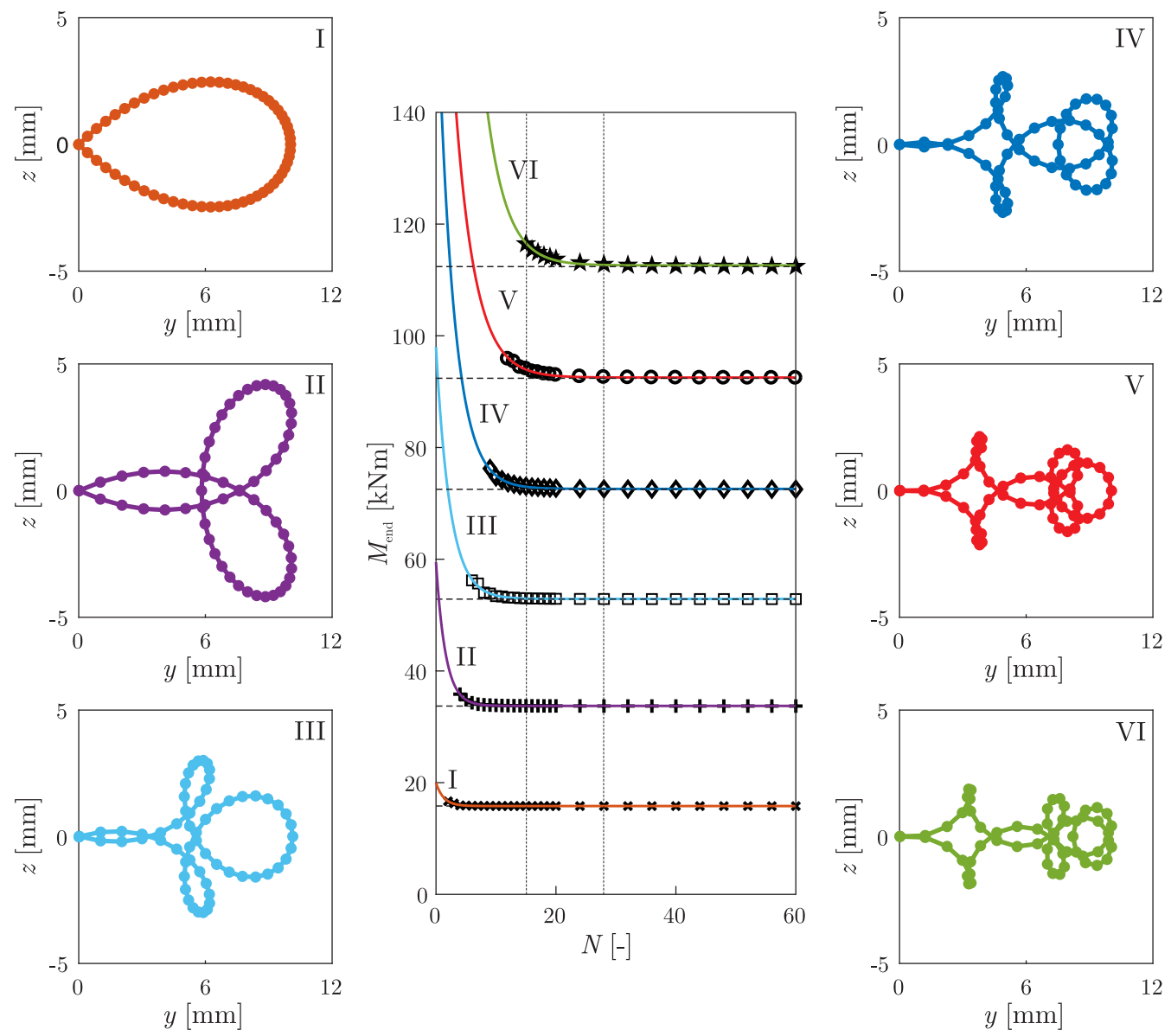

Figure 5: Sensitivity of predicting critical torsional buckling loads to number of FE elements used for first six buckling modes. Left and right panels depict the shapes of each mode on $y-z$ plane, while horizontal dashed lines indicate critical buckling loads predicted by Abaqus.

\section{Post-buckling analysis}

Following the successful validation of the developed FE, we now focus on analyzing the post-buckling behaviour of the rod depicted in Fig. 2(b). Following the analysis in Section 3, the rod is discretized also into $N=60 \mathrm{FE}$ elements and the load stepping method is used to compute the deformation of the rod subjected to an end torque $M_{e n d}$, varied in steps of $\Delta M_{e n d}=1 \mathrm{Nm}$. The simulation starts from $M_{e n d}=1 \mathrm{Nm}$ and with the straight configuration. Once, the solution for nodal displacements is found, it is used as a starting point for the subsequent step. An example of the post-buckling scenario computed in this way is shown in Figs 6 (a)-(f), where three displacements, $x_{31}, y_{31}$ and $z_{31}$ along $x, y$ and $z$ axes 
and three rotations, $\zeta_{x 31}, \zeta_{y 31}$ and $\zeta_{z 31}$ around $x, y$ and $z$ axes for the node corresponding to the middle point of the rod (node 31 ), are plotted as a function of the end torque $M_{\text {end }}$.

As mentioned before, the onset to torsional buckling can be traced by looking at any of the displacements $x_{31}, y_{31}$ and $z_{31}$ or rotations $\zeta_{y 31}$ and $\zeta_{z 31}$. As shown in Fig. 6, initially there is no visible change in the shape of the rod as the torque increases (orange segment), meaning that the straight configuration of the rod is preserved. This continues until the first critical torsional buckling load (I) is reached, where an abrupt change in rod's shape takes place. Now the rod takes a shape corresponding to the first critical torsional buckling mode (with one loop).

Unlike in Section 3, the post-buckling solution marked in black is continued until another abrupt change in any of the variables $\left(x_{31}, y_{31}, z_{31}, \zeta_{y 31}, \zeta_{z 31}\right)$ occurs. As can be seen by looking at the black curve, with the increasing $M_{\text {end }}$ the helix grows in size, but qualitatively the rod still has the same shape with one loop. An example of the rod's shape for $M_{\text {end }}=42955 \mathrm{Nm}$ is shown in the $3 \mathrm{D}$ plot at the bottom of Fig. 6. For $M_{e n d}=63472 \mathrm{Nm}$ a clear change is visible, when there is a sudden jump (marked by vertical arrow) to a new configuration (marked in red) and depicted in, which can easily be applied the second panel at the bottom of Fig. 6. The new configuration is a helix with two loops. Even though it has two loops, it is qualitatively a different configuration than the one corresponding to the II torsional mode described in Section 3.

As before, we continue increasing the end torque, observing that the amplitude of displacements varies continuously, until a further sudden change which occurs at $M_{\text {end }}=99430 \mathrm{Nm}$. This time the new branch is a helix with three loops (marked in blue), depicted in the 3D plot shown in the third panel at the bottom of Fig. 6, for $M_{\text {end }}=124774 \mathrm{Nm}$. A subsequent jump occurs at $M_{\text {end }}=141666 \mathrm{Nm}$, when the rod takes the shape of a helix with four loops (green curve). An example of this configuration for $M_{\text {end }}=145850 \mathrm{Nm}$, is depicted in the fourth panel at the bottom of Fig. 6. The analysis concludes when $M_{\text {end }}$ reaches $150000 \mathrm{Nm}$. As a verification of the above analysis, we repeat it in the backward direction, starting at $M_{\text {end }}=150000 \mathrm{Nm}$, using the last point from the forward analysis as an initial configuration and using the load step of $\Delta M_{\text {end }}=-1 \mathrm{Nm}$.

As in the previous case, abrupt changes in the shape of the rod (marked by vertical arrow) take place when the number of loops in the helix decrease with decreasing $M_{\text {end }}$. A hysteresis effect is observed, as transitions occur at different values of $M_{\text {end }}$. For example, the transition between the helix with four loops and helix with three loops takes place for $M_{\text {end }}=135850 \mathrm{Nm}$, while for forward analysis it occurs at $M_{\text {end }}=141666 \mathrm{Nm}$. This means that in the interval between $135850 \mathrm{Nm}$ and $141666 \mathrm{Nm}$, both helical configurations with four and three loops co-exist with each other. Similar co-existence is observed 
between the helical configurations with three and two loops in the interval between $96861 \mathrm{Nm}$ and 99430 $\mathrm{Nm}$, as well as between helical configurations with two and one loops in the interval between $57311 \mathrm{Nm}$ and $63472 \mathrm{Nm}$. From the analysis presented above, one can conclude that it is possible to identify regions where various stable helical configurations co-exist and a complete scenario of transitions between various helical configurations can be observed.

If the above analysis started from the straight configuration of the rod for $M_{\text {end }}$ after the first critical torsional buckling mode (I - 33714.4 Nm), one would see a slightly different buckling scenario as the rod would buckle at the load corresponding to mode II and take a shape shown in Fig. 4 (b). It is possible to follow this solution as before, until it loses stability and the rod bifurcates into the helical configuration with two loops observed before, from where the post-buckling scenario follows what is shown in Fig. 6 . The same happens if the other critical torsional buckling modes (III, IV, V or VI) are used as starting point. This means that no matter what is the initial state, in the post-buckling scenarios a helix with multiple loops is created. 

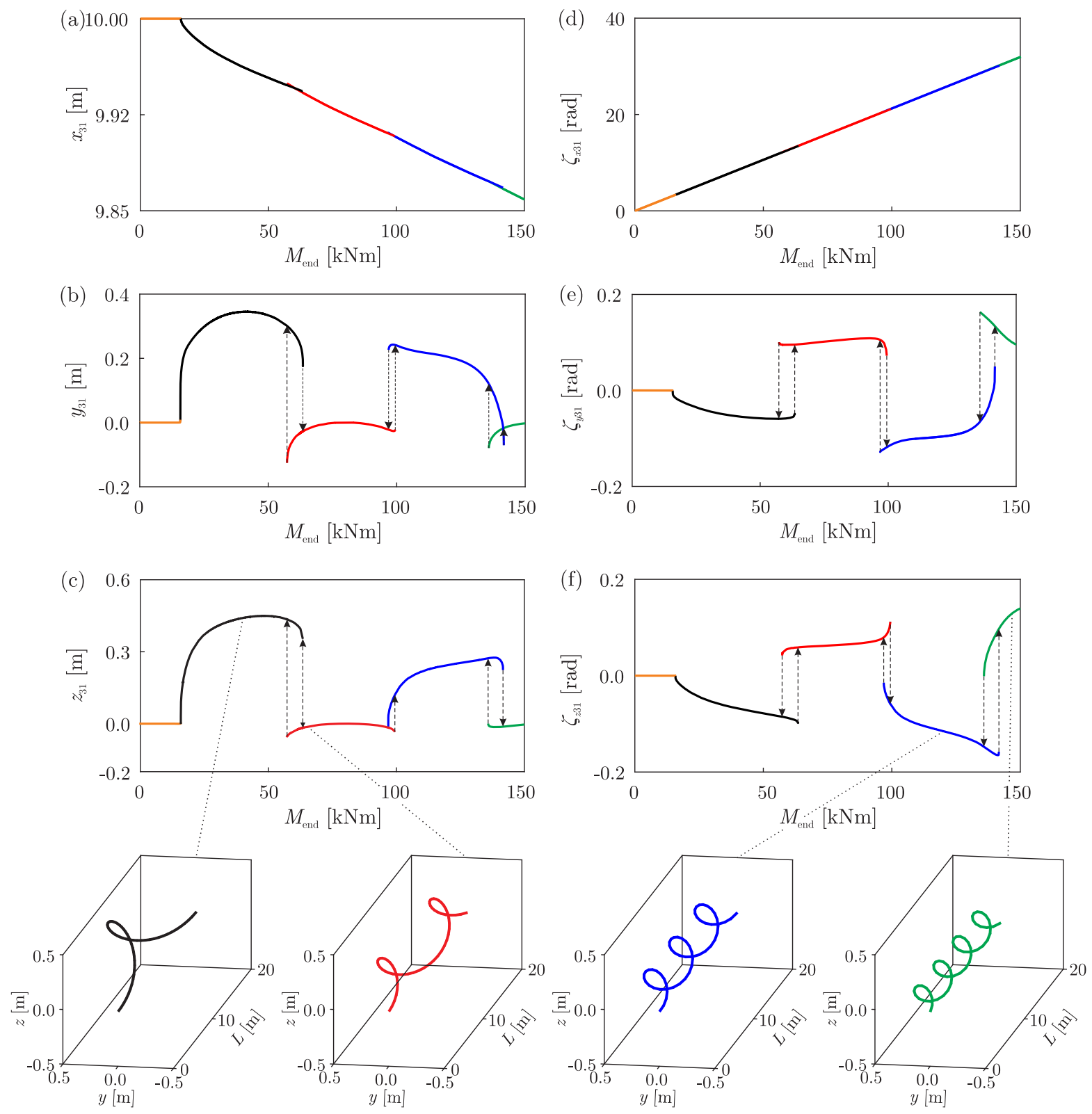

Figure 6: Post-buckling scenario showing displacements (a) $x_{31}$, (b) $y_{31}$, (c) $z_{31}$ and rotations (d) $\zeta_{x 31}$, (e) $\zeta_{y 31}$, (f) $\zeta_{z 31}$ of the middle node as a function of end torque $M_{\text {end }}$. Simulation is run in forward and backward directions revealing co-existence of various helical configurations, which examples are shown in bottom panels (from left to right) for $M_{\text {end }}$ : $42955 \mathrm{Nm}, 63472 \mathrm{Nm}, 124774 \mathrm{Nm}, 145850 \mathrm{Nm}$, respectively. 

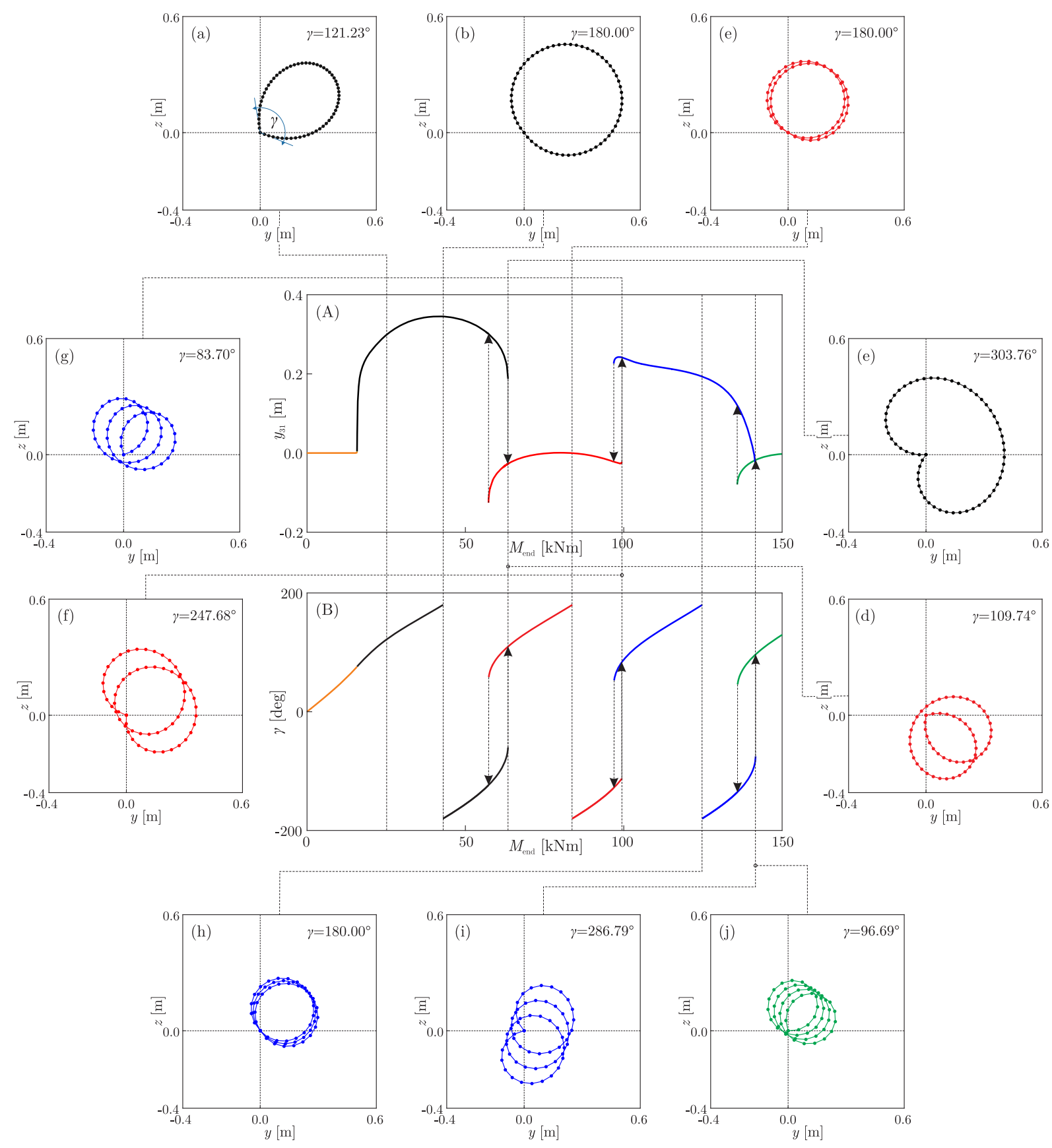

Figure 7: A detailed view into the post-buckling scenario, where panel (A) depicts lateral displacement $y_{31}$ and panel (B) depicts the change of angle $\gamma$ (defined in panel (a)) as a function of end torque $M_{\text {end }}$. A 2D projections of the shape of the rod on the y-z plane showing the evolution of the post-buckling branches are shown in panels (a) $M_{\text {end }}=25000 \mathrm{Nm}$, (b) $M_{\text {end }}=42955 \mathrm{Nm}$, (c) $M_{\text {end }}=63475 \mathrm{Nm}$, (d) $M_{\text {end }}=63475 \mathrm{Nm}$, (e) $M_{\text {end }}=83750 \mathrm{Nm}$, (f) $M_{\text {end }}=99430 \mathrm{Nm}$, (g) $M_{\text {end }}=99430 \mathrm{Nm},(\mathrm{h}) M_{\text {end }}=124774 \mathrm{Nm},(\mathrm{i}) M_{\text {end }}=141669 \mathrm{Nm},(\mathrm{j}) M_{\text {end }}=141669 \mathrm{Nm}$. 
The post-buckling scenario depicted in Fig. 6 can suggest that within the same branch there are no distinct changes in the shape of a helical configuration of the rod. To verify that, we look in detail into one of the lateral displacements of the middle node $y_{31}$, originally shown in Fig. 6(b), which zoomed in view is depicted in Fig. 7(A). As a start let us look at the projection of the shape of the rod on the $y-z$ plane for $M_{\text {end }}=25000 \mathrm{Nm}$, which constitutes part of the branch marked in black colour, shown in Fig. 7(a). Let us define an angle $\gamma$, which is an angle between the first and the last element on the $y-z$ plane, as shown in panel (a). Now, this angle is computed using arctan function (modulo $\pi$ ) for each loading step from the simulation depicted in Fig. 6, and plotted as a function of $M_{\text {end }}$ in Fig. 7(B). As it is shown, $\gamma$ changes between $\pm 180^{\circ}$.

Looking from the beginning of the considered interval of $M_{e n d}$, we see that $\gamma$ increases continuously with the end torque. No jumps in $\gamma$ are observed when the first critical torsional buckling load is passed. This continues until $M_{e n d}=42955 \mathrm{Nm}$, when the shape of the rod becomes almost circular on the $\mathrm{y}-\mathrm{z}$ plane, as shown in panel (b). As $M_{\text {end }}$ increases, we are still on the same branch (black), but there is a slight change in the shape of the helical configuration. Note, that $\gamma=180^{\circ}$ marks the onset to the transition into a further helical buckling configuration with two loops, as the second loop starts to develop. As $M_{\text {end }}$ increases the black branch becomes unstable and the rod bifurcates at $M_{\text {end }}=63475 \mathrm{Nm}$ from the helix with one loop, shown in panel (c), into helix with two loops, as shown in panel (d) (the new branch is marked in red). Following the red branch, we note that a similar change in shape occurs again when $\gamma=180^{\circ}$, (see panel (e)), when the rod takes almost circular shape. From that point onward a new loop starts to develop leading to a sudden transition to the new configuration of helix with three loops that occurs at $M_{\text {end }}=99430 \mathrm{Nm}$. This transition takes place between the configuration depicted in panels (f) and (g). Exactly the same change in the rod's shape occurs for the blue branch when $\gamma=180^{\circ}$ (see panel (h)), which again marks the onset when additional loop starts to develop, before a transition from the blue (see panel (i)) into the green branch (see panel (j)) takes place, for $M_{\text {end }}=141669 \mathrm{Nm}$.

The examples given in panels (c), (d), (f), (g), (i) and (f) of Fig. 7 correspond to the transitions between various helical configurations of the rod when the analysis is computed in the forward direction. As desribed before, when the analysis is conducted in a backward direction the transitions occur at different values of $M_{\text {end }}$, but the changes in the helical configuration indicated by $\gamma=180^{\circ}$ occur at the same loads. This time they mark the onset to unwinding of a loop, before the transition to the helix with fewer loops takes place.

Based on the analysis performed in this and previous sections, we can conclude that within the analysed interval of end torque $\left(M_{\text {end }} \in(0,150000) \mathrm{Nm}\right)$ there are 6 critical torsional buckling modes 
possible (depicted in Fig. 4) as well 4 configurations where the rod takes the shape of helix with multiple loops. The transitions between the helical configurations occur at different values of $M_{\text {end }}$, than the critical torsional buckling loads corresponding to modes I, II, III, IV, V and VI. As mentioned earlier, different torsional buckling modes can be used as starting points to compute the post-buckling scenarios. Nevertheless, after the buckling point we are able to follow a particular solution until it becomes unstable, when the rod bifurcates into one of the helical configurations described above and depicted in Fig. 8. The original post-buckling scenario is kept on the graph as well.

If one starts the analysis after the first critical buckling load from the straight configuration of the rod, the second critical torsional buckling mode (II), depicted in purple, can be captured. It becomes unstable at $M_{\text {end }}=54390 \mathrm{Nm}$, when the rod takes shape of a helix with one loop (black curve). This means that we revert to the original post-buckling scenario. Exactly the same happens for case III (cyan branch), IV (grey branch) and V (orange branch) buckling modes, when the transitions to helices with two (red branch) and three loops (blue branch) take place at $M_{\text {end }}=85590 \mathrm{Nm}$, at $M_{\text {end }}=97900 \mathrm{Nm}$ and $M_{\text {end }}=107632 \mathrm{Nm}$, respectively. Note, that both branches corresponding to modes IV and V revert to the same helical configuration (helix with three loops).

For the branch corresponding to mode VI (light green), the solution becomes unstable outside of the considered interval of $M_{\text {end }}$, whereas transition into a helix with four loops occurs at $M_{\text {end }}=162680 \mathrm{Nm}$. Inside the analysed region, there is an additional critical torsional buckling mode(VII, marked in light blue), occuring at $M_{\text {end }}=132535 \mathrm{Nm}$. Following this branch, we observe that it becomes unstable quite quickly at $M_{\text {end }}=138696 \mathrm{Nm}$, when the rod takes shape of a helix with four loops. In this particular case, a rapid transition might be related to number of elements used in this case, causing the transition to happen earlier than expected. Examples of 3D views of the rod's configuration for the branches corresponding to modes II-VII are shown in Figs 8(a)-(f), respectively. The analysis presented above suggests that the helical configurations are much more stable than the configurations corresponding to the critical torsional buckling modes.

Throughout this study it was proven that by using the derived FE beam element, we obtained a reliable tool allowing us to precisely determine the critical torsional (as well as others) buckling loads and compute the full post-buckling scenarios, as well as to pinpoint the transitions between various helical configurations. Performing such an analysis in commercial software such as ABAQUS requires much more computational time as well larger number of more complex elements. As an example a test run in ABAQUS was performed to compute the responses of the rod subjected to an end torque from interval $M_{\text {end }} \in(0,24000) \mathrm{Nm}$. The simulation is done utilising STATIC simulation with stabilisation, 
with 4224 hexagonal elements, including geometric nonlinearities (NLGEOM ON). Using this approach one can get a similar response to the one obtained using the newly developed FE beam element, but the computational time of $4 \mathrm{hr} 38 \mathrm{~min} 12 \mathrm{~s}$ required is considerably longer than when using our approach which took 10 min $21 \mathrm{~s}$. Moreover, the simulation in ABAQUS was carried out utilizing 10 CPUs on High Performance Computer at University of Aberdeen, while the simulation utilising the beam element presented in this paper was done using just $1 \mathrm{CPU}$ and code in Matlab on a standard machine. This demonstrates a clear advantage of the new FE capable to calculate precisely the torsional post-buckling scenarios and to gain a much needed insight into the intricacies behind the mechanics of helical buckling. 

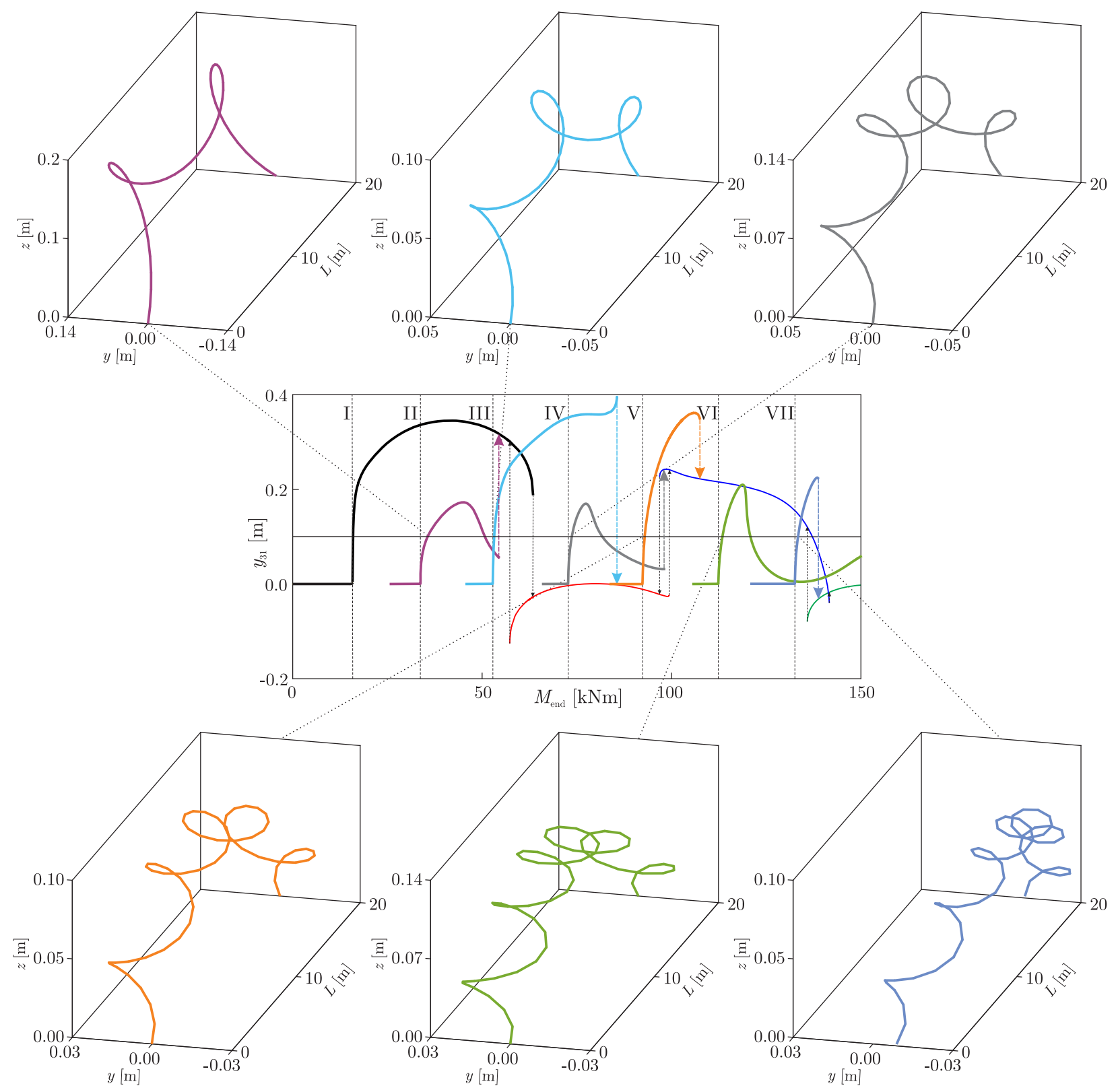

Figure 8: Post-buckling scenario computed using different critical buckling modes as a starting point (I, II, III, IV, V, VI, VII marked in black, purple, cyan, grey, orannge, light green and light blue respectively). Note, that once the path corresponding to particular buckling mode, the rod takes helical configuration, described in detail in Fig. 6. Examples of 3D views of the shape of the rod for: (a) $M_{\text {end }}=35600 \mathrm{Nm}$, (b) $M_{\text {end }}=53180 \mathrm{Nm}$, (c) $M_{\text {end }}=71480 \mathrm{Nm}$, (d) $M_{\text {end }}=92952 \mathrm{Nm}$, (e) $M_{\text {end }}=113490 \mathrm{Nm}$, (f) $M_{\text {end }}=132535 \mathrm{Nm}$. 


\section{Concluding remarks}

In this paper we have introduced a new nonlinear FE beam element derived to study the mechanics of helical buckling of rods with axi-symmetric cross-sections. The element is based on Euler-Bernoulli beam element, though it takes into account geometrical nonlinearities associated with axial, lateral and torsional DOFs and nonlinear coupling between them. The stiffness characteristics of the beam element are derived from the strain energy, which involves a careful treatment of nonlinear strains. An inclusion of the nonlinear terms in axial and shear strains allows to obtain a fully nonlinear geometric stiffness matrix of the beam element, where all the DOFs are coupled with each other.

The details of the derivation were given in Section 2, where a particular attention is paid to the selection of specific nonlinear components in the strain energy to fully describe the buckling couplings between various DOFs. As a next step, a careful validation of the proposed beam element was performed to determine its predictive capabilities in calculating the critical torsional buckling loads. This involves static analysis of a rod, subjected to a gradually increasing end torque, which allows to pinpoint the exact onset to helical buckling. The results obtained in this way were compared with the ones computed using commercial FE package Abaqus. As shown in Section 3, a very good agreement between those approaches was obtained for first six critical torsional buckling loads considered. As a next step we performed a series of tests to determine the sensitivity of critical torsional buckling load prediction to number of FE elements used. As shown in Fig. 5, only $28 \mathrm{FE}$ elements were required to accurately predict first six critical buckling modes, which is significantly less than in Abaqus.

Once, the validation of the proposed FE beam element has been completed, we focussed our attention on analysing the post buckling scenarios of a rod subjected to the end torque. The post-buckling behaviour is carefully monitored, by varying the end torque and computing corresponding static deformations of the rod. In this way we are able to observe various torsional buckling configurations, study their evolution and co-existence of various configurations. As shown in Fig. 8, starting from the initial straight configuration the rod buckles helically following the first critical buckling mode (helix with one loop), but as the load increases there is a bifurcation into another state (helix with two loops). Subsequent bifurcations into helices with three and four loops takes place as the end torque increase. An important observation is that the bifurcations into helices with multiple loops take place at different values of end torque than those corresponding to principal critical torsional buckling modes (I-VI, depicted in Fig. 5).

Additionally, it was possible to identify regions where two different helical configurations of the rod co-exist with each other, for the same value of $M_{\text {end }}$. As shown in Fig. 7 , it is possible to exactly pinpoint the onset to the transition between various helices with multiple loops, by monitoring the changes in angle 
$\gamma$ (as defined in Fig. 7 (a)). This shows that the transition to another helical configuration starts to occur way before the actual bifurcation point. We also verified if the post-buckling scenario depends on the initial configuration by using different critical buckling modes (II-VII) as starting points for the postbuckling analysis (see Fig. 8). It appears that the post-buckling scenario of helices with multiple loops is dominant, as in each case once the configuration corresponding to modes II-VII becomes unstable, the rod reverts to the one of the original scenario (see Fig. 6).

Considering the robust validation of the proposed nonlinear beam element for slender rods reported in this paper, we can conclude that a robust mathematical model allowing investigate bifurcations in helical buckling and post-buckling scenarios of slender twisted rods have been developed. Moreover, the proposed approach allows for considerable savings in computational time, when compared to a similar analysis computed by a commercial FE software, while maintaining high computation accuracy to study complexities of post-buckling scenarios. Due to its universality, the proposed method can be applied to various engineering and science problems. Currently the authors are incorporating the proposed FE element into a full dynamical FE model of a drill-string assembly and its experimental validation on the versatile drilling rig [34].

\section{Acknowledgments}

The authors would like to acknowledge the use of the Maxwell High Performance Computing Cluster of the University of Aberdeen IT Service and various industrial projects sponsored by Oil \& Gas Innovation Centre (OGIC), which stipulated these studies.

\section{References}

[1] S. Goyal, N. Perkins, and C. Lee, "Nonlinear dynamics and loop formation in Kirchhoff rods with implications to the mechanics of DNA and cables," Journal of Computational Physics, vol. 209, no. 1, pp. $371-389,2005$.

[2] X. Tan and P. Digby, "Buckling of drill string under the action of gravity and axial thrust," International Journal of Solids and Structures, vol. 30, no. 19, pp. 2675 - 2691, 1993.

[3] C. Sun and S. Lukasiewicz, "A new model on the buckling of a rod in tubing," Journal of Petroleum Science and Engineering, vol. 50, no. 1, pp. 78-82, 2006. 
[4] Z. Liang and Z. Zhu, "Critical helical buckling load assessment of coiled tubing under axial force by use of the explicit finite-element method," Journal of Petroleum Science and Engineering, vol. 169, pp. 51-57, 2018.

[5] Y. Z. Liu and J. W. Zu, "Stability and bifurcation of helical equilibrium of a thin elastic rod," Acta Mechanica, vol. 167, no. 1, pp. 29-39, 2004.

[6] M. Liangruksa, T. Laomettachit, and S. Wongwises, "Theoretical study of DNA's deformation and instability subjected to mechanical stress," International Journal of Mechanical Sciences, vol. 130, pp. $324-330,2017$.

[7] J. M. T. Thompson and A. R. Champneys, "From helix to localized writhing in the torsional postbuckling of elastic rods," Proceedings of the Royal Society of London. Series A: Mathematical, Physical and Engineering Sciences, vol. 452, no. 1944, pp. 117-138, 1996.

[8] J. M. T. Thompson, M. Silveira, G. H. M. van der Heijden, and M. Wiercigroch, "Helical postbuckling of a rod in a cylinder: with applications to drill-strings," Proceedings of the Royal Society A: Mathematical, Physical and Engineering Science, 2012.

[9] Y. Miyazaki and K. Kondo, "Analytical solution of spatial elastica and its application to kinking problem," International Journal of Solids and Structures, vol. 34, no. 27, pp. 3619 - 3636, 1997.

[10] Y. Z. Liu and Y. Xue, "Stability analysis of helical rod based on exact Cosserat model," Applied Mathematics and Mechanics, vol. 32, no. 5, p. 603, 2011.

[11] A. H. Nayfeh and S. A. Emam, "Exact solution and stability of postbuckling configurations of beams," Nonlinear Dynamics, vol. 54, no. 4, pp. 395-408, 2008.

[12] D. J. Braun, "On the optimal shape of compressed rotating rod with shear and extensibility," International Journal of Non-Linear Mechanics, vol. 43, no. 2, pp. 131 - 139, 2008.

[13] S. Stoykov and P. Ribeiro, "Nonlinear forced vibrations and static deformations of 3D beams with rectangular cross section: The influence of warping, shear deformation and longitudinal displacements," International Journal of Mechanical Sciences, vol. 52, no. 11, pp. 1505 - 1521, 2010. Special Issue on Non-linear Oscillations.

[14] M. R. Barnes, S. Adriaenssens, and M. Krupka, "A novel torsion/bending element for dynamic relaxation modeling," Computers \& Structures, vol. 119, pp. 60 - 67, 2013. 
[15] E. Sapountzakis and V. Mokos, "Dynamic analysis of 3D beam elements including warping and shear deformation effects," International Journal of Solids and Structures, vol. 43, no. 22, pp. 6707 -6726, 2006.

[16] Y. B. Yang and W. McGuire, "Stiffness Matrix for Geometric Nonlinear Analysis," Journal of Structural Engineering, vol. 112, no. 4, pp. 853-877, 1986.

[17] C. Meier, A. Popp, and W. A. Wall, "A locking-free finite element formulation and reduced models for geometrically exact Kirchhoff rods," Computer Methods in Applied Mechanics and Engineering, vol. 290 , pp. $314-341,2015$.

[18] S. Sen and S. Awtar, "A Closed-Form Nonlinear Model for the Constraint Characteristics of Symmetric Spatial Beams," ASME Journal of Mechanical Design, vol. 135, no. 3, 2013.

[19] R. Sampaio, M. Piovan, and G. V. Lozano, "Coupled axial/torsional vibrations of drill-strings by means of non-linear model," Mechanics Research Communications, vol. 34, no. 56, pp. 497-502, 2007.

[20] M. Trindade and R. Sampaio, "Dynamics of beams undergoing large rotations accounting for arbitrary axial rotations," J. Guid. Control Dynam, vol. 25, no. 4, pp. 634-643, 2002.

[21] C. Germay, V. Denoäl, and E. Detournay, "Multiple mode analysis of the self-excited vibrations of rotary drilling systems," Journal of Sound and Vibration, vol. 325, no. 1-2, pp. 362-381, 2009.

[22] H. Al-Naser and Y. Khulief, "Finite element dynamic analysis of drillstrings," Finite Elements in Analysis and Design, vol. 41, no. 13, pp. 1270-1288, 2005.

[23] Y. Khulief, F. Al-Sulaiman, and S. Bashmal, "Vibration analysis of drillstrings with self-excited stick-slip oscillations," Journal of Sound and Vibration, vol. 299, no. 3, pp. 540 - 558, 2007.

[24] T. Ritto, C. Soize, and R. Sampaio, "Non-linear dynamics of a drill-string with uncertain model of the bit-rock interaction," International Journal of Non-Linear Mechanics, vol. 44, pp. 865-876, 2009.

[25] V. Gulyayev and N. Shlyun, "Global analysis of drill string buckling in the channel of a curvilinear bore-hole," Journal of Natural Gas Science and Engineering, vol. 40, pp. 168 - 178, 2017.

[26] M. Paidoussis, T. Luu, and S. Prabhakar, "Dynamics of a long tubular cantilever conveying fluid downwards, which then flows upwards around the cantilever as a confined annular flow," Journal of Fluids and Structures, vol. 24, no. 11, pp. 111-128, 2007. 
[27] A. Jafari, R. Kazemi, and M. F. Mahyari, "The effects of drilling mud and weight bit on stability and vibration of a drill string," Journal of Vibration and Acoustics, Transactions of the ASME, vol. 134, no. $1,2012$.

[28] J. S. Przemieniecki, Theory of matrix structural analysis. Dover Publications, 2012.

[29] M. Kapitaniak, V. Vaziri, and M. Wiercigroch, "Helical buckling of thin rods: FE modelling," MATEC Web Conf., vol. 211, p. 02010, 2018.

[30] O. C. Zienkiewicz, The Finite Element Method. McGraw-Hill, 1977.

[31] J. F. Doyle, Nonlinear Structural Dynamics Using FE Methods. Cambridge University Press, 2014.

[32] A. Bazoune, Y. Khulief, and N. Stephen, "Shape Functions of Three-Dimensional Timoshenko Beam E," Journal of Sound and Vibration, vol. 259, no. 2, pp. 473 - 480, 2003.

[33] DS Simulia, Abaqus Analysis User's Manual 6.12.

[34] M. Kapitaniak, V. Vaziri Hamaneh, J. Páez Chávez, K. Nandakumar, and M. Wiercigroch, "Unveiling Complexity of Drill-String Vibration: Experiments and Modelling," International Journal of Mechanical Sciences, vol. 101-102, pp. 324-337, 2015. 